\title{
Comparação dos parâmetros bioquímicos entre gestação única e gemelar de ovelhas da raça Dorper durante gestação, parto e pós-parto
}

\author{
[Comparison of biochemical parameters between single and twin pregnancies of \\ Dorper breed ewes during gestation, lambing and postpartum] \\ B.P. Santarosa ${ }^{1}$, G.N. Dantas ${ }^{1}$, D.O.L. Ferreira ${ }^{2}$, B. Santos ${ }^{1}$, R.K. Takahira ${ }^{1}$, \\ M.G. Carvalho ${ }^{1}$, A.A. Silva ${ }^{3}$, R.C. Gonçalves ${ }^{1}$ \\ ${ }^{1}$ Universidade Estadual Paulista - Botucatu, SP \\ ${ }^{2}$ Secretaria da Agricultura e Abastecimento do Estado de São Paulo - Agudos, SP \\ ${ }^{3}$ Universidade Federal Rural do Rio de Janeiro - Seropédica, RJ
}

\author{
B.P. Santarosa \\ https://orcid.org/0000-0003-0937-1919 \\ G.N. Dantas \\ https://orcid.org/ 0000-0002-1271-1171 \\ D.O.L. Ferreira \\ https://orcid.org/0000-0002-2227-0621 \\ B. Santos \\ https://orcid.org/0000-0002-5285-7597 \\ R.K. Takahira \\ https://orcid.org/0000-0003-3323-4199 \\ M.G. Carvalho \\ https://orcid.org/0000-0001-9507-3787 \\ A.A. Silva $=$ \\ https://orcid.org/0000-0002-4254-5856 \\ R.C. Gonçalves \\ https://orcid.org/0000-0002-0076-6159
}

\section{RESUMO}

Nas ovelhas, a demanda de nutrientes aumenta durante a gestação, especialmente nas últimas seis semanas, quando ocorre maior desenvolvimento do feto. Os objetivos deste trabalho foram estudar o perfil bioquímico de ovelhas durante a gestação e no periparto imediato, comparando-se gestação única com gemelar. Foram utilizadas 60 ovelhas, Dorper, divididas em dois grupos experimentais com base no diagnóstico de gestação ultrassonográfico: grupo 1: 30 ovelhas com feto único; grupo 2: 30 ovelhas com gestação gemelar. Em nove momentos de coleta, foram mensurados no soro sanguíneo: ureia, creatinina, proteína total (PT), albumina, globulina, aspartato aminotransferase (AST), gamaglutamiltransferase, fosfatase alcalina, colesterol e triglicérides. O peso vivo das ovelhas do G2 foi maior que do G1 em todos os momentos, inclusive quando estavam vazias. Houve diferença entre as ovelhas do G1 e do G2 nos valores de PT, albumina, AST e triglicérides. A partir de 120 dias de gestação, o perfil proteico se modificou, com menores níveis de ureia, PT, albumina e globulina, mostrando que a demanda metabólica se intensificou no último mês da gestação. Os níveis de colesterol e triglicérides diminuíram de 140 dias de gestação para o parto. Este trabalho mostrou que a gestação proporcionou mudanças significativas no metabolismo da ovelha, principalmente no terço final da gestação, quando há maior necessidade de acompanhamento para evitar a incidência de doenças metabólicas.

Palavras-chave: bioquímica sérica, doenças metabólicas, toxemia da prenhez, ovinocultura

\begin{abstract}
In sheep, nutrient demand increases during gestation, particularly in the last six weeks, when the fetuses develop. The objectives of this study were to evaluate the biochemical profile of ewes during pregnancy and in the immediate peripartum comparing single with twin pregnancies. Sixty ewes of Dorper breed were divided into two groups by pregnancy ultrasonographic diagnosis: 1 - 30 ewes with single fetus gestation; 2 - 30 ewes with twin pregnancy. In nine collection moments the following parameters were measured: urea, creatinine, total protein (TP), albumin, globulin, aspartate aminotransferase, gamma glutamyltransferase, alkaline phosphatase, cholesterol and triglycerides. The weight of G2 sheep was higher than G1 since they were empty. There were differences in G1 and G2 sheep metabolism in TP, Albumin, AST and Triglycerides. From 120 days of pregnancy, the protein profile changed, with lower levels of urea, TP, albumin and globulin, showing that the metabolic demand intensified in the last month of pregnancy. The levels of cholesterol and triglycerides decreased from 140 days of pregnancy to lambing day. This study showed that gestation provided significant changes in the ewe's metabolism, especially in the final third of gestation, when there is a greater need to follow the animals to avoid the incidence of metabolic diseases.
\end{abstract}

Keywords: biochemical profile, metabolic diseases, pregnancy toxaemia, sheep

Recebido em 28 de março de 2018

Aceito em 9 de outubro de 2018

E-mail: biancasantarosavet@gmail.com 


\section{INTRODUÇÃO}

Nas ovelhas, a demanda de nutrientes aumenta durante a gestação, particularmente nas últimas seis semanas, quando ocorre maior desenvolvimento do feto, que alcança aproximadamente $70 \%$ do seu crescimento, além do requerimento de nutrientes para $o$ desenvolvimento mamário. $\mathrm{O}$ período entre o final da gestação e o início da lactação tem sido considerado o estágio de maior interesse do ciclo produtivo. Esse período é acompanhado de diversas alterações anatômicas, hormonais e metabólicas devido à preparação para o parto e o início da lactação. Essas modificações no perfil metabólico de ovelhas podem predispor a ocorrência de transtornos metabólicos (Bani Ismail et al., 2008). Entre as enfermidades de maior ocorrência nesse período, destaca-se a toxemia da prenhez (TP), que acomete ovelhas e cabras, principalmente no último mês de gestação (Campos et al., 2010; Souto et al., 2013; Lima et al., 2015).

O monitoramento dos padrões proteico, energético e mineral em ovinos é uma ferramenta de grande importância para a adequação alimentar e da condição metabólica de ovelhas, considerando-se a pressão do processo de intensificação da produtividade, que, em muitos casos, promove os desequilíbrios entre o ingresso e o egresso dos nutrientes, tornando-se, assim, um entrave para a produção animal (Santos et al., 2012; Oliveira et al., 2014).

Na literatura, os estudos sobre a TP ocorrem de forma crescente, porém não há trabalhos que discutam as alterações no perfil metabólico e bioquímico em ovelhas Dorper durante toda a prenhez, o parto e o pós-parto imediato, comparando-se gestações única e gemelar. Portanto, diante da alta relevância dessa doença na espécie ovina devido à alta mortalidade, este trabalho estudou conjuntamente diversas variáveis do metabolismo da ovelha prenhe, a fim de conhecer o comportamento desses parâmetros ao longo da gestação e compará-los entre gestação única e gemelar, e com isso identificar alterações que possam estabelecer diagnóstico precoce dessa enfermidade.

\section{MATERIAL E MÉTODOS}

Os métodos adotados no presente trabalho foram aprovados pela Comissão de Ética no Uso de Animais (Ceua) da Faculdade de Medicina Veterinária e Zootecnia (FMVZ) da Universidade Estadual Paulista (Unesp), Campus de Botucatu (Protocolo 189/2014).

Foram selecionadas 150 ovelhas vazias, hígidas, da raça Dorper, com dois a cinco anos de idade, que participaram de protocolo de sincronização de estro. Empregou-se o protocolo de longa duração (12 dias): o implante vaginal com $0,33 \mathrm{~g}$ de progesterona (Cidr®, Zoetis) foi colocado no dia 0 e permaneceu por 12 dias (D0 a D12). Após a remoção dele, foram administrados, em cada animal, 400 a 500UI de gonadotrofina coriônica equina (eCG) (Novormon ${ }^{\circledR}$, Zoetis) por via intramuscular. Passadas 48 horas (D14), foi feita a inseminação artificial em tempo fixo (IATF) por laparoscopia, com sêmen congelado, e realizaram-se as coletas do primeiro momento, considerado controle (MI). Depois de 30 dias (MG30), procedeu-se ao diagnóstico de gestação única ou gemelar com ultrassom portátil (My LabTM30 Vet Gold Esaote ${ }^{\circledR}$, Esaote Healthcare do Brasil, São Paulo, SP, Brasil), com o transdutor linear com frequência de $5,0 \mathrm{MHz}$, para o exame transretal, quando se definiu o grupo experimental em que a ovelha foi incluída. Foram delineados dois grupos experimentais com base no diagnóstico ultrassonográfico de gestação: grupo 1: 30 ovelhas, com feto único; grupo 2: 30 ovelhas, com gestação gemelar. Os animais vazios foram excluídos do lote experimental. Para os demais momentos, foi empregado o transdutor convexo com frequência de $3,5 \mathrm{MHz}$, para avaliação da viabilidade fetal. Todas as ovelhas receberam vermífugo após o parto (Monepantel, Zolvix®, Novartis Saúde Animal) e vacina polivalente contra clostridioses (Sintoxan-T Polivalente $\AA$, Merial) aos 30 e 60 dias antes do parto.

As ovelhas foram soltas pela manhã e ficaram a pasto durante o dia. A pastagem utilizada foi o capim vaquero (cultivar Cynodon dactylon). No final da tarde, os animais foram presos em baias coletivas de $12 \mathrm{~m}^{2}$, em barracão de alvenaria, com 
cama de palha de arroz, onde foram alimentados com $0,5 \mathrm{~kg} /$ animal de ração para manutenção e $1,0 \mathrm{~kg} /$ animal de silagem de milho. No barracão havia disponibilidade ad libitum de sal mineral comercial para ovinos (Ovinofós com Monensina ${ }^{\circledR}$, Tortuga Companhia Zootécnica Agrária, Mairinque, SP, Brasil) em cochos de alvenaria cobertos. A água foi proveniente de poço artesiano localizado na própria cabanha e era disponível em cochos automáticos de forma ad libitum. A dieta total fornecida pela propriedade aos animais foi submetida à análise bromatológica (realizada pelo Laboratório de Bromatologia - Departamento de Nutrição e Melhoramento Animal FMVZ/Unesp-Botucatu) e dos minerais (realizada pelo Instituto Campineiro de Análise de Solo e Adubo Campinas, SP) da ração, da silagem de milho e da pastagem, conforme está descrito nas Tab. 1 e 2.

Tabela 1. Análise bromatológica da ração, da silagem de milho e do capim vaquero utilizados no experimento. Resultados expressos em $100 \%$ de matéria seca

\begin{tabular}{lccccccccccc}
\hline \multicolumn{1}{c}{ Amostra } & $\%$ MS & $\%$ PB & $\%$ EE & $\%$ Minerais & $\%$ FDN & $\%$ FDA & $\begin{array}{c}\% \\
\text { Hemicelulose }\end{array}$ & $\mathrm{pH}$ & $\begin{array}{c}\text { Energia } \\
\text { cal/g }\end{array}$ & Celulose & Lignina \\
\hline Ração & 88,45 & 20,85 & 9,10 & 6,27 & 14,58 & 5,05 & 9,53 & - & 3871 & - & - \\
Silagem de milho & 27,83 & 8,28 & 3,20 & 3,84 & 50,55 & 29,51 & 21,04 & 3,53 & - & - & - \\
Capim vaquero & 26,19 & 13,48 & 5,94 & 6,59 & 76,39 & 33,75 & 42,64 & - & - & 23,89 & 7,46 \\
\hline
\end{tabular}

MS - matéria seca; PB - proteína bruta; EE - extrato etéreo; FB - fibra bruta; FDN - fibra em detergente neutro; FDA - fibra em detergente ácido.

Tabela 2. Análise dos minerais da ração, da silagem de milho e do capim vaquero utilizados no experimento. Resultados expressos em matéria seca (ppm)

\begin{tabular}{lcccccccccccccccc}
\hline \multicolumn{1}{c}{ Amostra } & $\mathrm{N}$ & $\mathrm{P}$ & $\mathrm{K}$ & $\mathrm{Ca}$ & $\mathrm{Mg}$ & $\mathrm{S}$ & $\mathrm{Fe}$ & $\mathrm{Mn}$ & $\mathrm{Cu}$ & $\mathrm{Zn}$ & $\mathrm{Na}$ & $\mathrm{B}$ & $\mathrm{Co}$ & $\mathrm{Mo}$ & $\mathrm{Al}$ \\
\hline $\begin{array}{l}\text { Ração* } \\
\begin{array}{l}\text { Silagem de } \\
\text { milho }\end{array}\end{array}$ & - & 7290 & 6250 & 10920 & 2260 & 3370 & 250 & 120 & 35 & 200 & 5230 & 190 & 1 & 12 & 130 \\
$\begin{array}{l}\text { Capim } \\
\text { vaquero }\end{array}$ & 14160 & 1670 & 13410 & 2960 & 2520 & 1350 & 271,5 & 46,5 & 6 & 20 & 74,5 & 2,6 & $<0,3$ & $<0,3$ & 413 \\
\hline
\end{tabular}

*Umidade $85^{\circ}$ : 11,40\%; umidade $110^{\circ}$ : 11,77\%; matéria orgânica: 92,11\%; cinzas: 7,89\%.

Os momentos experimentais foram definidos como: MI - imediatamente após a IATF (controle); MG30 - 30 dias de gestação; MG90 90 dias de gestação; MG120 - 120 dias de gestação; MG130 - 130 dias de gestação; MG140 - 140 dias de gestação; MP - dia do parto; MPP1 - 24 horas após o parto; MPP2 - 48 horas após o parto. Em todos os momentos, foram realizados os seguintes procedimentos, antes da soltura dos animais para a pastagem, de forma padronizada: exame físico para aferição das funções vitais (frequências cardíaca e respiratória, movimentos ruminais e temperatura retal); pesagem em balança (Coimma ${ }^{\circledR}$ - Comércio e Indústria de Madeiras e Metalúrgica São Cristóvão Ltda., Dracena, SP); ultrassonografia transabdominal para confirmação da viabilidade fetal e coleta de amostras de sangue total.

Todas as amostras de sangue total pela punção da veia jugular foram coletadas pela manhã, com os animais em jejum alimentar, dos dois grupos experimentais e nos nove momentos. Foram utilizadas agulhas (BD Vacutainer®, BD Medical, Curitiba, PR, Brasil) 30x0,8mm, tubos a vácuo, com gel ativador de coágulo e sem anticoagulante. Após a retração do coágulo, a amostra foi centrifugada para obtenção de soro a $2.000 \mathrm{x} \mathrm{g}$, durante 10 minutos (Centrífuga Combate Celm ${ }^{\circledR}$ Cia. Equipadora de Laboratórios Modernos, Barueri, SP, Brasil). Foram separadas alíquotas de 2,0mL de soro em tubos plásticos (Eppendorf do Brasil Ltda., São Paulo,SP, Brasil), as quais foram armazenadas em freezer $-20^{\circ} \mathrm{C}$. Em uma única vez, as amostras foram descongeladas e analisadas em espectrofotômetro (Cobas Mira Plus - Roche®) pelo Laboratório de Patologia Clínica Veterinária da FMVZ/Unesp-Botucatu. Foram mensuradas, no soro sanguíneo das ovelhas, nos diferentes momentos, pelo método enzimático colorimétrico, as concentrações de: colesterol, triglicérides, ureia, proteína total, albumina, aspartato aminotransferase (AST), gamaglutamiltransferase (GGT) e fosfatase 
alcalina (FA); e pelo método cinético colorimétrico: creatinina.

Para análise estatística, foi utilizado o software SigmaStat 3.5. Para todos os parâmetros das análises bioquímicas e o peso vivo, foi realizada análise de medidas repetidas no tempo (One Way Repeated Measures Anova) para comparação dos momentos de avaliação dentro de cada grupo individualmente. Quando houve significância $(\mathrm{P}<0,05)$, foi realizado o teste de Tukey para comparação das médias $(\mathrm{P}<0,05)$. Também em cada momento, foi realizado o teste $\mathrm{t}$ de Student para comparação dos grupos em estudo $(\mathrm{P}<0,05)$. Para os dados de período gestacional e do peso ao nascimento dos cordeiros, empregou-se apenas estatística descritiva.

\section{RESULTADOS E DISCUSSÃO}

A média e o desvio-padrão do período gestacional das 60 ovelhas foi de 144,75 $\pm 2,12$ dias de gestação, variando de 140 a 149 dias. A média do peso ao nascimento dos 90 cordeiros foi de $3,29 \pm 0,94 \mathrm{~kg}$, sendo 42 fêmeas e 48 machos. Houve necessidade de realização de cesariana em apenas uma ovelha com gestação simples, devido à distocia de causa materna por não haver dilatação cervical. Dos 59 partos normais, 51 foram auxiliados, sendo distocia fetal a causa mais comum. A maioria das ovelhas pariu no amanhecer ou anoitecer; apenas cinco ovelhas pariram no pasto durante o dia.
Nenhuma ovelha apresentou sintomatologia clínica sugestiva de TP ou outra enfermidade, portanto nenhum tratamento foi instituído na propriedade onde foram realizadas as coletas das amostras.

A pesagem (Tab. 3) foi realizada somente até o MP, pois não haveria disparidade em $24 \mathrm{~h}$ e $48 \mathrm{~h}$ após o parto. Além disso, evitou-se transportar as ovelhas com cordeiros ao pé até o centro de manejo, onde se localizava a balança, para evitar acidentes. O G2 sempre apresentou média maior que o G1, devido à gestação de dois cordeiros. No entanto, a diferença foi observada desde o momento da IATF, o que pode ser justificado pelo fato de que as ovelhas com maior peso corporal apresentaram, provavelmente, maior taxa de ovulação e, portanto, maior taxa de prolificidade comparadas às ovelhas do G1. Isso foi comprovado por Boucinhas et al. (2006), que descreveram que o sistema de manejo em que as ovelhas foram suplementadas três semanas antes e quatro após o início do manejo para concepção, três semanas antes do parto e durante a lactação influenciou positivamente o peso, a condição corporal, a fertilidade e a prolificidade. Ao longo dos momentos, ambos os grupos apresentaram médias diferentes entre si, sendo o maior valor encontrado em MG140. No MP, notou-se perda de peso expressiva devido ao parto, pela subtração do(s) cordeiro(s), dos envoltórios fetais, do líquido amniótico e do alantoide.

Tabela 3. Peso vivo $(\mathrm{kg})$ das ovelhas dos dois grupos experimentais (G1 e G2) nos diferentes momentos (M) de coleta

\begin{tabular}{lccc}
\cline { 2 - 3 } \multicolumn{1}{c}{ M } & \multicolumn{2}{c}{ Grupos } & \multirow{2}{*}{$\mathrm{P}$} \\
\cline { 2 - 3 } & $\mathrm{G} 1$ & $\mathrm{G} 2$ & 0,006 \\
MI & $45,6 \pm 6,5 \mathrm{~dB}$ & $54,1 \pm 7,7 \mathrm{dA}$ & $<0,001$ \\
MG30 & $52,5 \pm 6,7 \mathrm{cB}$ & $60,6 \pm 7,8 \mathrm{dA}$ & $<0,001$ \\
MG120 & $61,8 \pm 7,9 \mathrm{bB}$ & $71,0 \pm 8,3 \mathrm{bcA}$ & $<0,001$ \\
MG130 & $63,1 \pm 7,6 \mathrm{bB}$ & $71,4 \pm 8,4 \mathrm{bcA}$ & $<0,001$ \\
MG140 & $65,3 \pm 7,8 \mathrm{abB}$ & $74,2 \pm 7,7 \mathrm{abA}$ & $<0,001$ \\
MP & $67,6 \pm 8,0 \mathrm{aB}$ & $76,4 \pm 7,8 \mathrm{aA}$ & 0,008 \\
P & $61,7 \pm 8,5 \mathrm{bB}$ & $68,3 \pm 8,6 \mathrm{cA}$ & \\
\hline
\end{tabular}

Dados apresentados como média \pm desvio-padrão. ${ }^{\text {a,b }}$ Médias seguidas pela mesma letra minúscula na coluna não diferiram estatisticamente pelo teste de Tukey $(\mathrm{P}>0,05) .{ }^{\mathrm{A} . \mathrm{B}}$ Médias seguidas por letras maiúsculas diferentes na linha diferiram estatisticamente pelo teste $\mathrm{t}(\mathrm{P}<0,05)$.

As médias dos valores de ureia e creatinina (Tab. 4) não se diferenciaram estatisticamente entre os grupos em nenhum dos momentos de coleta, porém apresentaram diferença entre os momentos dentro de cada grupo estudado. Os resultados das médias da ureia estiveram dentro da normalidade para a espécie ovina, exceto nos momentos MG30 e MG120 em ambos os grupos. 
Oliveira et al. (2014) avaliaram três grupos de ovelhas com diferentes estágios do período gestacional (início: 50 a 60 dias, meio: 80 a 90 dias, e fim: 130 a 140 dias) e também notaram níveis mais elevados de ureia, porém relacionados com o metabolismo proteico e não em razão da alteração de função renal. Os valores da ureia no $\mathrm{G} 1$ e no $\mathrm{G} 2$ foram maiores no MG120, e decaíram progressivamente até o MPP2. A ocorrência de redução de metabólitos proteicos com o avanço da gestação ou da lactação está associada ao balanço proteico negativo, devido ao maior crescimento dos cordeiros e da produção de leite (Silva et al., 2013).

Tabela 4. Concentrações séricas de ureia e creatinina $(\mathrm{mg} / \mathrm{dL})$ das ovelhas dos dois grupos experimentais (G1 e G2) nos diferentes momentos (M) de coleta

\begin{tabular}{lcccccc}
\hline \multirow{2}{*}{ M } & \multicolumn{3}{c}{ Ureia } & \multicolumn{3}{c}{ Creatinina } \\
\cline { 2 - 7 } & $\mathrm{G} 1$ & $\mathrm{G} 2$ & $\mathrm{P}$ & $\mathrm{G} 1$ & $\mathrm{G} 2$ & $\mathrm{P}$ \\
\hline MI & $41,9 \pm 6,5 \mathrm{~b}$ & $42,6 \pm 6,8 \mathrm{bc}$ & 0,711 & $0,98 \pm 0,14 \mathrm{~cd}$ & $0,99 \pm 0,10 \mathrm{bc}$ & 0,913 \\
MG30 & $45,8 \pm 7,8 \mathrm{ab}$ & $45,6 \pm 4,9 \mathrm{a}$ & 0,881 & $1,04 \pm 0,13 \mathrm{c}$ & $1,06 \pm 0,12 \mathrm{~b}$ & 0,711 \\
MG90 & $32,0 \pm 7,3 \mathrm{c}$ & $29,8 \pm 4,7 \mathrm{ef}$ & 0,225 & $1,23 \pm 0,18 \mathrm{a}$ & $1,18 \pm 0,14 \mathrm{a}$ & 0,281 \\
MG120 & $50,0 \pm 9,7 \mathrm{a}$ & $49,8 \pm 8,1 \mathrm{a}$ & 0,953 & $1,19 \pm 0,16 \mathrm{a}$ & $1,25 \pm 0,09 \mathrm{a}$ & 0,135 \\
MG130 & $41,6 \pm 5,5 \mathrm{~b}$ & $42,3 \pm 5,2 \mathrm{c}$ & 0,641 & $0,95 \pm 0,13 \mathrm{~d}$ & $0,97 \pm 0,12 \mathrm{bc}$ & 0,559 \\
MG140 & $35,3 \pm 7,8 \mathrm{c}$ & $35,2 \pm 6,0 \mathrm{de}$ & 0,957 & $0,95 \pm 0,14 \mathrm{~cd}$ & $1,02 \pm 0,11 \mathrm{~b}$ & 0,349 \\
MP & $32,6 \pm 7,8 \mathrm{c}$ & $33,0 \pm 6,8 \mathrm{ef}$ & 0,835 & $1,06 \pm 0,18 \mathrm{~b}$ & $1,06 \pm 0,19 \mathrm{~b}$ & 0,931 \\
MPP1 & $31,1 \pm 7,3 \mathrm{c}$ & $32,0 \pm 11,5 \mathrm{ef}$ & 0,724 & $1,00 \pm 0,14 \mathrm{~cd}$ & $1,05 \pm 0,19 \mathrm{~b}$ & 0,349 \\
MPP2 & $30,4 \pm 8,9 \mathrm{c}$ & $27,9 \pm 9,1 \mathrm{f}$ & 0,412 & $0,95 \pm 0,14 \mathrm{~d}$ & $0,91 \pm 0,14 \mathrm{c}$ & 0,253 \\
P & $<0,001$ & $<0,001$ & & $<0,001$ & $<0,001$ & \\
\hline
\end{tabular}

Dados apresentados como média \pm desvio-padrão. ${ }^{\mathrm{a}, \mathrm{b}}$ Médias seguidas pela mesma letra minúscula na coluna não diferiram estatisticamente pelo teste de Tukey $(\mathrm{P}>0,05)$. Ureia: 17,12 a $42,80 \mathrm{mg} / \mathrm{dL}$; creatinina 1,2 a $1,9 \mathrm{mg} / \mathrm{dL}$ (Kaneko et al., 2008).

A creatinina esteve dentro ou abaixo do intervalo de referência (Kaneko et al., 2008) em todos os momentos em ambos os grupos. A mesma situação foi descrita por Santos et al. (2012) e Silva et al. (2013) em ovelhas Santa Inês. Isso mostrou que a gestação única ou gemelar não influenciou na função renal das ovelhas estudadas.

As médias da proteína total sérica (Tab. 5) no G1 no MPP1 e em ambos os grupos no momento MPP2 ficaram abaixo da faixa de normalidade para ovinos. Todas as médias de albumina (Tab. 5) ficaram dentro do intervalo de referência. Os valores médios de globulina (Tab. 5) ficaram abaixo da normalidade em ambos os grupos do momento MG130 até o MPP2. Nos resultados das médias de albumina e globulina, houve diferença entre os momentos em ambos os grupos estudados.

No Rio Grande do Sul, Ribeiro et al. (2004), ao observarem ovelhas em pastoreio, encontraram redução da $\mathrm{PT}$ e da albumina com o avanço da gestação e da lactação, provavelmente pela baixa ingestão de proteína durante essa fase. Já Oliveira et al. (2014) descreveram níveis de metabolismo proteico dentro da normalidade durante as três fases gestacionais de ovelhas Santa Inês, provavelmente devido ao consumo proteico de 396g/dia de proteína, em média. De acordo com o NRC (Nutrient..., 2007), ovelhas no final da gestação possuem requerimento de $193 \mathrm{~g} / \mathrm{PB} /$ dia. No presente estudo, as ovelhas consumiram, em média, 400g/dia de proteínas (312g PB/dia provenientes da ração e $82,8 \mathrm{~g} /$ dia provenientes da silagem de milho), o que é acima do recomendado para as três fases de gestação.

A albumina é considerada o indicador mais sensível para determinar o estado nutricional proteico (Oliveira et al., 2014). No presente trabalho, os níveis de albumina refletiram o consumo adequado de proteína e até acima do recomendado, embora tenha atingido média próxima do limite inferior nos momentos pósparto (MPP1 e MPP2). 
Tabela 5. Concentrações séricas de proteína total, albumina e globulina ( $\mathrm{g} / \mathrm{dL}$ ) das ovelhas dos dois grupos experimentais (G1 e G2) nos diferentes momentos (M) de coleta

\begin{tabular}{|c|c|c|c|c|c|c|c|c|c|}
\hline \multirow{2}{*}{ M } & \multicolumn{3}{|c|}{ Proteína total } & \multicolumn{3}{|c|}{ Albumina } & \multicolumn{3}{|c|}{ Globulina } \\
\hline & G1 & G2 & $\mathrm{P}$ & G1 & $\mathrm{G} 2$ & $\mathrm{P}$ & G1 & G2 & $\mathrm{P}$ \\
\hline MI & $6,5 \pm 0,5 b$ & $6,7 \pm 0,6 \mathrm{~cd}$ & 0,135 & $2,8 \pm 0,2 \mathrm{cdB}$ & $3,1 \pm 0,2 \mathrm{bA}$ & $<0,001$ & $3,7 \pm 0,4 b$ & $3,6 \pm 0,5 \mathrm{~cd}$ & 0,591 \\
\hline MG90 & $7,1 \pm 0,5 \mathrm{a}$ & $7,1 \pm 0,5 b c$ & 0,938 & $3,0 \pm 0,3 b c$ & $3,1 \pm 0,2 b$ & 0,153 & $4,1 \pm 0,4 \mathrm{a}$ & $4,0 \pm 0,5 \mathrm{ab}$ & 0,281 \\
\hline MG120 & $7,3 \pm 0,9 a$ & $7,5 \pm 0,4 \mathrm{a}$ & 0,389 & $3,2 \pm 0,5 \mathrm{ab}$ & $3,4 \pm 0,3 \mathrm{a}$ & 0,051 & $4,1 \pm 0,7 \mathrm{a}$ & $4,1 \pm 0,4 \mathrm{a}$ & 0,770 \\
\hline MG140 & $6,1 \pm 0,9 b$ & $6,4 \pm 0,4 \mathrm{de}$ & 0,238 & $2,8 \pm 0,4 \mathrm{cdB}$ & $3,0 \pm 0,2 \mathrm{bA}$ & 0,011 & $3,3 \pm 0,6 \mathrm{cde}$ & $3,3 \pm 0,3 \mathrm{def}$ & 0,897 \\
\hline MP & $6,1 \pm 0,9 b$ & $6,4 \pm 0,4 \mathrm{de}$ & 0,240 & $2,8 \pm 0,5 \mathrm{~dB}$ & $3,0 \pm 0,3 \mathrm{bA}$ & 0,023 & $3,3 \pm 0,6 \mathrm{~cd}$ & $3,3 \pm 0,3 \mathrm{def}$ & 0,875 \\
\hline MPP1 & $5,6 \pm 0,7 \mathrm{cB}$ & $6,0 \pm 0,5 \mathrm{fA}$ & 0,049 & $2,6 \pm 0,4 \mathrm{eB}$ & $2,8 \pm 0,2 \mathrm{cdA}$ & 0,003 & $3,1 \pm 0,4 \mathrm{e}$ & $3,1 \pm 0,3 \mathrm{ef}$ & 0,635 \\
\hline
\end{tabular}

Dados apresentados como média \pm desvio-padrão. ${ }^{\mathrm{a}, \mathrm{b}}$ Médias seguidas pela mesma letra minúscula na coluna não diferiram estatisticamente pelo teste de Tukey $(\mathrm{P}>0,05)$. ${ }^{\text {A.B }}$ Médias seguidas por letras maiúsculas diferentes na linha diferiram estatisticamente pelo teste $\mathrm{t}(\mathrm{P}<0,05)$. Proteína total: 6 a 7,9g/dL; albumina: 2,4 a 4,0g/dL; globulina: 3,5 a $5,7 \mathrm{~g} / \mathrm{dL}$ (Kaneko et al., 2008).

A ureia é sintetizada no fígado em quantidades proporcionais à concentração de amônia produzida no rúmen e sua concentração está diretamente relacionada aos níveis proteicos da dieta (Brondani et al., 2016). Ribeiro et al. (2003) encontraram, durante o ano, níveis séricos médios de ureia de $37,97 \mathrm{mg} / \mathrm{dL}$ em borregas da raça Corriedale, enquanto Rabassa et al. (2009) obtiveram, em ovelhas, durante o outono/inverno, níveis médios de $30,68 \mathrm{mg} / \mathrm{dL}$. Segundo Ribeiro et al. (2004), em ovelhas Border Leicester $\times$ Texel que apresentaram queda no valor da condição corporal do início ao final da gestação (3,30 e 2,11, respectivamente), os teores de albumina sanguínea também diminuíram na metade e no final desse período (31,05 e 24,44g/L, respectivamente). Contudo, os teores de ureia sérica não diferiram significativamente, sendo de $5,87 \mathrm{mmol} / \mathrm{L}$ para as ovelhas na metade da gestação e de $5,59 \mathrm{mmol} / \mathrm{L}$ para as ovelhas em final de gestação. Segundo os autores, isso se deve ao fato de as ovelhas em final de gestação possuírem demanda proteica maior para o crescimento fetal e o desenvolvimento do úbere, fazendo com que diminuam os teores de albumina, o que não ocorre com os teores de ureia, pelo fato de esta exprimir diretamente a concentração de amônia no rúmen. Apesar disso, no presente estudo, os níveis de ureia diminuíram a partir do MG140 em ambos os grupos. Antunovic et al. (2011) observaram menores concentrações de ureia
(mmol/L), em ovelhas gestantes que em ovelhas lactantes $(5,70$ e 6,80, respectivamente $)$ e maiores de albumina (g/L) (30,93 e 28,60, respectivamente), e atribuíram esse resultado à maior captação de albumina pelo organismo para a produção de colostro e leite.

Os valores de globulina apresentaram queda a partir do MG130, conjuntamente às concentrações de PT, devido à colostrogênese, e transferência de imunoglobinas, principalmente IgG, para o(s) cordeiro(s) pela mamada, conferindo fonte essencial de imunidade passiva pelos anticorpos maternos (Ulian et al., 2014). A mesma situação foi observada por Alencar e colabodores (2007), em ovelhas das raças Suffolk e Ideal, e Oliveira et al. (2016), em Santa Inês, que apresentaram redução da globulina, uma vez que grande quantidade de imunoglobulinas deixou o plasma durante os meses finais de gestação, para produção de colostro na glândula mamária. A mobilização da reserva proteica durante a lactação pode resultar também em redução das globulinas, semelhante àquela observada na gestação (Alencar et al., 2007).

As enzimas hepáticas (Tab. 6) estiveram dentro da faixa de normalidade para ovinos na maioria dos momentos em ambos os grupos, exceto para a AST no G1 nos momentos MPP1 e MPP2; a GGT no MG120 para ambos os grupos e no 
MPP2 para o G2. O aumento de AST observado pode estar relacionado com a maior atividade da musculatura do útero no parto, com o decúbito ou com o aumento do metabolismo hepático, decorrente principalmente da mobilização de reservas corporais frequente nesse período (Nascimento et al., 2015). Esses níveis elevados de GGT ocorreram devido à mobilização de reservas para a lactação (Nascimento et al., 2015), porém ficaram muito próximos ao limite superior. Diferentemente desse estudo, Santos et al. (2012) não encontraram diferença entre os momentos analisados (30 e sete dias pré-parto; no parto; às $24 \mathrm{~h}, 72 \mathrm{~h}$, aos cinco, 15 e 30 dias pós-parto, em ovelhas Santa Inês, para as enzimas AST, FA e GGT.

Tabela 6. Concentrações séricas de AST, FA e GGT (UI/L) das ovelhas dos dois grupos experimentais (G1 e G2) nos diferentes momentos (M) de coleta

\begin{tabular}{|c|c|c|c|c|c|c|c|c|c|}
\hline \multirow{2}{*}{ M } & \multicolumn{3}{|c|}{ AST } & \multicolumn{2}{|c|}{ FA } & \multicolumn{4}{|c|}{ GGT } \\
\hline & G1 & $\mathrm{G} 2$ & $\mathrm{P}$ & G1 & $\mathrm{G} 2$ & $\mathrm{P}$ & G1 & G2 & $\mathrm{P}$ \\
\hline MI & $146,0 \pm 36,4 \mathrm{bB}$ & $177,9 \pm 56,5 \mathrm{abA}$ & 0,018 & $180,7 \pm 75,8 \mathrm{a}$ & $173,5 \pm 87,6 \mathrm{a}$ & 0,756 & $45,9 \pm 12,7 \mathrm{~b}$ & $45,9 \pm 9,6 \mathrm{~b}$ & 0,992 \\
\hline MG30 & $164,5 \pm 32,2 b$ & $176,9 \pm 46,0 \mathrm{abc}$ & 0,263 & $155,2 \pm 76,6 \mathrm{a}$ & $134,2 \pm 72,6 b$ & 0,327 & $48,3 \pm 12,6 b$ & $46,2 \pm 7,6 b$ & 0,480 \\
\hline MG90 & $168,7 \pm 35,6 b$ & $163,0 \pm 34,1 \mathrm{bc}$ & 0,570 & $105,1 \pm 41,8 \mathrm{bc}$ & $104,6 \pm 50,5 \mathrm{bcd}$ & 0,969 & $49,1 \pm 9,9 \mathrm{~b}$ & $45,5 \pm 8,2 b$ & 0,171 \\
\hline MG120 & $184,8 \pm 45,0 \mathrm{~b}$ & $189,8 \pm 38,6 \mathrm{ab}$ & 0,678 & $124,1 \pm 47,3 b$ & $116,4 \pm 62,6 \mathrm{bc}$ & 0,615 & $59,2 \pm 16,3 \mathrm{a}$ & $56,9 \pm 9,7 \mathrm{a}$ & 0,546 \\
\hline MG130 & $138,1 \pm 26,2 b$ & $142,3 \pm 30,7 b c$ & 0,601 & $96,9 \pm 34,7 \mathrm{bcd}$ & $89,4 \pm 49,3 \mathrm{~cd}$ & 0,530 & $47,4 \pm 10,6 b$ & $45,5 \pm 7,4 \mathrm{~b}$ & 0,477 \\
\hline MG140 & $137,0 \pm 29,4 b$ & $136,5 \pm 23,9 \mathrm{c}$ & 0,952 & $95,8 \pm 42,4 \mathrm{bcd}$ & $83,7 \pm 38,8 \mathrm{~cd}$ & 0,299 & $47,6 \pm 10,5 b$ & $46,3 \pm 8,2 b$ & 0,651 \\
\hline MP & $148,9 \pm 44,3 b$ & $141,6 \pm 33,7 b c$ & 0,522 & $73,6 \pm 34,9 \mathrm{~d}$ & $75,9 \pm 35,6 \mathrm{~d}$ & 0,816 & $46,9 \pm 10,8 b$ & $46,5 \pm 8,8 b$ & 0,910 \\
\hline MPP1 & $328,4 \pm 137,1 \mathrm{aA}$ & $220,0 \pm 100,4 \mathrm{abB}$ & 0,003 & $100,8 \pm 54,0 \mathrm{bcd}$ & $91,7 \pm 41,9 \mathrm{~cd}$ & 0,518 & $45,0 \pm 10,8 b$ & $46,0 \pm 7,9 b$ & 0,772 \\
\hline MPP2 & $288,2 \pm 121,5 \mathrm{a}$ & $226,5 \pm 109,1 \mathrm{a}$ & 0,067 & $78,9 \pm 41,0 \mathrm{~cd}$ & $86,5 \pm 46,3 \mathrm{~cd}$ & 0,535 & $50,0 \pm 9,8 b$ & $53,1 \pm 9,1 b$ & 0,247 \\
\hline $\mathrm{P}$ & $<0,001$ & $<0,001$ & & $<0,001$ & $<0,001$ & & $<0,001$ & $<0,001$ & \\
\hline
\end{tabular}

Dados apresentados como média \pm desvio-padrão. ${ }^{\mathrm{a}, \mathrm{b}}$ Médias seguidas pela mesma letra minúscula na coluna não diferiram estatisticamente pelo teste de Tukey $(\mathrm{P}>0,05)$. ${ }^{\mathrm{A} B \mathrm{~B}}$ Médias seguidas por letras maiúsculas diferentes na linha diferiram estatisticamente pelo teste $\mathrm{t}(\mathrm{P}<0,05)$. AST: 60 a 280UI/L; FA: 68 a 387UI/L; GGT 20 a 52UI/L (Kaneko et al., 2008).

As médias do colesterol (Tab. 7) ficaram acima dos valores de referência para ovinos nos momentos MG30, MG120 ao MP, em ambos os grupos. Para o triglicérides, o mesmo resultado ocorreu do momento MG30 ao MP. Além de alterações na condição fisiológica, como a gestação e a lactação, outros fatores afetam o metabolismo energético nos animais, como o manejo alimentar e a inclusão de ingredientes com alto teor de lipídios na dieta (Fernandes et al., 2012). No caso das ovelhas, somente a ração fornecida continha 9,10\% MS em $\mathrm{EE}$, o que significa alto teor de gordura. Portanto, os níveis elevados de colesterol e triglicérides podem ser justificados também pela nutrição desses animais.

Ao contrário deste estudo, vários autores descreveram que as ovelhas com gestação gemelar apresentaram maiores valores de colesterol e triglicérides do que as ovelhas de gestação única no terço final da prenhez (Hamadeh et al., 1996; Balikci et al., 2009; Raoofi et al., 2013). Esses autores atribuíram esse fato à resistência à insulina, que ocorre de forma fisiológica no final da gestação, pois esse hormônio influencia diretamente no metabolismo do tecido adiposo durante a gestação. Além disso, a diminuição da capacidade de resposta dos tecidos alvos à insulina durante a gestação tardia predispõe as ovelhas a apresentarem níveis mais elevados de colesterol, triglicérides e lipoproteínas. No entanto, neste trabalho não foi observada diferença nas concentrações de colesterol entre os dois grupos e, no caso dos triglicérides, as ovelhas com gestação gemelar apresentaram níveis mais baixos do que aquelas que pariram somente um cordeiro no último momento antes do parto e no parto. 
Tabela 7. Concentrações séricas de colesterol e triglicérides $(\mathrm{mg} / \mathrm{dL})$ das ovelhas dos dois grupos experimentais (G1 e G2) nos diferentes momentos (M) de coleta

\begin{tabular}{ccccccc}
\hline \multirow{2}{*}{ M } & \multicolumn{3}{c}{ Colesterol } & \multicolumn{3}{c}{ Triglicérides } \\
\cline { 2 - 7 } & $\mathrm{G} 1$ & $\mathrm{G} 2$ & $\mathrm{P}$ & $\mathrm{G} 1$ & $\mathrm{G} 2$ & $\mathrm{P}$ \\
\hline MI & $67,6 \pm 16,6 \mathrm{de}$ & $74,7 \pm 12,4 \mathrm{~cd}$ & 0,097 & $18,1 \pm 6,2 \mathrm{e}$ & $18,1 \pm 4,2 \mathrm{c}$ & 0,958 \\
MG30 & $82,6 \pm 18,4 \mathrm{ab}$ & $80,3 \pm 13,8 \mathrm{bc}$ & 0,631 & $28,8 \pm 9,8 \mathrm{cde}$ & $24,1 \pm 5,5 \mathrm{c}$ & 0,051 \\
MG90 & $70,6 \pm 10,6 \mathrm{~cd}$ & $70,2 \pm 8,8 \mathrm{de}$ & 0,876 & $35,3 \pm 12,3 \mathrm{bc}$ & $35,0 \pm 11,5 \mathrm{~b}$ & 0,935 \\
MG120 & $87,0 \pm 17,7 \mathrm{c}$ & $90,5 \pm 16,4 \mathrm{a}$ & 0,473 & $48,0 \pm 18,8 \mathrm{a}$ & $43,8 \pm 12,8 \mathrm{a}$ & 0,370 \\
MG130 & $76,9 \pm 14,0 \mathrm{a}$ & $78,0 \pm 11,4 \mathrm{bcd}$ & 0,570 & $42,4 \pm 15,8 \mathrm{ab}$ & $37,2 \pm 10,3 \mathrm{ab}$ & 0,187 \\
MG140 & $84,1 \pm 16,2 \mathrm{ab}$ & $84,1 \pm 11,5 \mathrm{ab}$ & 0,996 & $45,1 \pm 24,1 \mathrm{aA}$ & $31,9 \pm 8,4 \mathrm{bB}$ & 0,017 \\
MP & $76,8 \pm 14,2 \mathrm{bc}$ & $78,0 \pm 14,5 \mathrm{bcd}$ & 0,776 & $33,3 \pm 12,0 \mathrm{bcdA}$ & $24,2 \pm 5,4 \mathrm{cB}$ & 0,002 \\
MPP1 & $69,3 \pm 11,6 \mathrm{~cd}$ & $73,7 \pm 11,5 \mathrm{~cd}$ & 0,184 & $23,8 \pm 10,5 \mathrm{de}$ & $21,0 \pm 6,9 \mathrm{c}$ & 0,280 \\
MPP2 & $60,9 \pm 11,2 \mathrm{e}$ & $63,4 \pm 15,5 \mathrm{e}$ & 0,504 & $22,2 \pm 11,1 \mathrm{e}$ & $21,9 \pm 6,4 \mathrm{c}$ & 0,887 \\
P & $<0,001$ & $<0,001$ & & $<0,001$ & $<0,001$ & \\
\hline
\end{tabular}

Dados apresentados como média \pm desvio-padrão. ${ }^{\mathrm{a}, \mathrm{b}}$ Médias seguidas pela mesma letra minúscula na coluna não diferiram estatisticamente pelo teste de Tukey $(\mathrm{P}>0,05)$. ${ }^{\text {A.B }}$ Médias seguidas por letras maiúsculas diferentes na linha diferiram estatisticamente pelo teste $\mathrm{t}(\mathrm{P}<0,05)$. Colesterol: 52 a $76 \mathrm{mg} / \mathrm{dL}$; triglicérides: 9 a 30mg/dL (Kaneko et al., 2008).

À semelhança do presente trabalho, Balikci et al. (2009) relataram aumento gradual no colesterol e de triglicérides séricos durante a gestação (aos 100 e 150 dias) quando se comparou aos 60 dias de prenhez e aos 45 dias pós-parto, em ovelhas que pariram um e dois cordeiros. Hamadeh et al. (1996) relataram concentrações séricas de colesterol maiores em ovelhas prenhes em relação às ovelhas vazias. O mesmo ocorreu neste estudo, em que a concentração de colesterol e triglicérides no momento MI se assemelhou aos níveis do pós-parto. Silva et al. (2013) observaram menores níveis de colesterol e triglicérides em ovelhas Santa Inês hígidas 10 dias antes do parto em relação aos 15 dias pósparto. No entanto, Özpınar e Firat (2003) relataram que os níveis de colesterol plasmático não foram significativamente diferentes para ovelhas prenhes e vazias. Os dados contraditórios descritos por esses autores podem ser justificados pela diferença no manejo nutricional de cada estudo ou na propriedade analisada, visto que é um fator que influencia diretamente nos níveis desses parâmetros.

Apesar das alterações encontradas nos parâmetros bioquímicos das ovelhas ao longo da gestação, não houve manifestação TP nas ovelhas estudadas devido ao manejo nutricional instituído na propriedade, que foi suficiente para suprir as necessidades de manutenção da saúde dos animais durante o período de gestação, parto e pós-parto.

\section{CONCLUSÕES}

Embora não tenha ocorrido TP nos animais estudados, este trabalho mostrou que a gestação proporcionou mudanças significativas no metabolismo da ovelha, principalmente no terço final da gestação, quando há maior necessidade de acompanhamento dos animais para evitar a incidência de doenças metabólicas.

\section{REFERÊNCIAS}

ALENCAR, N.X.; KOHAYAGAWA, A.; RODRIGUES, C.F.C. et al. Proteinograma e exame coproparasitológico de ovelhas das raças Ideal e Suffolk durante o periparto. Rev. Bras. Cienc. Vet., v.14, p.111-116, 2007.

ANTUNOVIC, Z.; NOVOSELEC, J.; SAUERWEIN, $\mathrm{H}$. et al. Blood metabolic profile and some of hormones concentration in ewes during different physiological status. Bulg. J. Agric. Sci., v.17, p.687695, 2011.

BALIKCI, E.; YILDIZ, A.; GURDOGAN, F. Investigation on some biochemical and clinical parameters for pregnancy toxemia in Akkaraman ewes. J. Anim. Vet. Adv., v.8, p.1268-1273, 2009.

BANI ISMAIL Z.; AL-MAJALI, A.M.; AMIREH F.; AL-RAWASHDEH O.F. Metabolic profiles in goat does in late pregnancy with and without subclinical pregnancy toxaemia. Vet. Clin. Pathol., v.37, p.434437, 2008.

BOUCINHAS, C.C.; SIQUEIRA, E.R.; MAESTÁ, S.A. Dinâmica do peso e da condição corporal e eficiência reprodutiva de ovelhas da raça Santa Inês e mestiças Santa Inês-Suffolk submetidas a dois sistemas de alimentação em intervalos entre partos de oito meses. Ciênc. Rural, v.36, p.904-909, 2006. 
BRONDANI, W.C.; LEMES, J.S.; FERREIRA, O.G.L. et al. Perfil metabólico de ovelhas em gestação. Arch. Zootec., v.65, p.1-6, 2016.

CAMPOS, A.G.; AFONSO, J.A.B.; SANTOS, R.A. et al. Estudo clínico-laboratorial da toxemia da prenhez em ovelhas: análise retrospectiva. Cienc. Anim. Bras., v.11, p.623-628, 2010.

FERNANDES, S.R.; FREITAS, J.A.; SOUZA, D.F. et al. Lipidograma como ferramenta na avaliação do metabolismo energético em ruminantes. Bras. Agrociênc., v.18, p.21-32, 2012.

HAMADEH, M.E.; BOSTEDT, H.; FAILING, K. Concentration of metabolic parameters in the blood of heavily pregnant and nonpregnant ewes. Berl. Munch. Tierarztl., v.109, p.81-86, 1996.

KANEKO, J.J.; HARVEY, J.W.; BRUSS, M.L. Clinical biochemistry of domestic animals. 6.ed. San Diego: Academic, 2008. 916p.

LIMA, E.H.F.; SOUTO, R.J.C.; SILVA, S.T.G. et al. Avaliação do perfil hematológico, bioquímico e lácteo em ovelhas gestantes suplementadas com monensina sódica. Vet. Zootec., v.22, p.634-650, 2015.

NASCIMENTO, P.M.; MORGADO, A.A.; NUNES, G.R. et al. Metabolismo oxidativo e perfil bioquímico de ovelhas Santa Inês no período periparto: efeito da suplementação parenteral com vitamina E. Semin. Ciênc. Agrár., v.36, p.1397-1408, 2015.

NUTRIENT requirements of small ruminants: sheep, goats, cervids and new world camelids. Washington: National Academy Press, 2007. 362p.

OLIVEIRA, R.P.M.; ASSANTE, R.T.; SILVA, A.F. et al. Avaliação do perfil metabólico em diferentes fases do periparto de ovelhas Santa Inês na Amazônia Ocidental. Rev. Bras. Saúde Prod. Anim., v.17, p.3744, 2016.

OLIVEIRA, R.P.M.; MADURO, A.H.P.; LIMA, E.S.; OLIVEIRA, F.F. Perfil metabólico de ovelhas Santa Inês em diferentes fases de gestação criadas em sistema semi-intensivo no estado do Amazonas. Ciênc. Anim. Bras., v.15, p.81-86, 2014.
ÖZPINAR, A.; FIRAT, A. Metabolic profile of prepregnancy, pregnancy and early lactation in multiple lambing sakı ewes 2. Changes in plasma progesterone, estradiol-17ß and cholesterol levels. Ann. Nutr. Metab., v.47, p.139-143, 2003.

RABASSA, V.R.; TABELEÃO, V.C.; SCHNEIDER, A. et al. Avaliação metabólica de ovelhas de cria mantidas em campo nativo durante o período de outono/inverno. Rev. Bras. Agrociênc., v.15, p.125$128,2009$.

RAOOFI, A.; JAFARIAN, M.; SAFI, S; VATANKHAH, M. Fluctuations in energy-related metabolites during the peri-parturition period in LoriBakhtiari ewes. Smal Ruminant Res., v.109, p.64-68, 2013.

RIBEIRO, L.A.O.; GONZÁLEZ, F.H.D.; CONCEIÇÃO, T.R. et al. Perfil metabólico de borregas Corriedale em pastagem nativa do Rio Grande do Sul. Acta Sci. Vet., v.31, p.167-170, 2003.

RIBEIRO, L.A.O.; MATTOS, R.C.; GONZÁLEZ, F.H. et al. Perfil metabólico de ovelhas Border Leicester x Texel durante a gestação e lactação. Rev. Port. Ciênc. Vet., v.99, p.155-159, 2004.

SANTOS, R.A.; CAMPOS, A.G.S.S.; AFONSO, J.A.B. et al. Efeito da administração de propileno glicol e cobalto associado à vitamina B12 sobre o perfil metabólico e a atividade enzimática de ovelhas da raça Santa Inês no periparto. Pesqui. Vet. Bras., v.32, Supl.1, p.60-66, 2012.

SILVA, J.S.C.; GUARANÁ, E.L.S.; LEMOS, V.F. et al. Metabolismo energético, proteico e mineral de ovelhas Santa Inês hígidas e com mastite subclínica. Pesqui. Vet. Bras., v.33, p.1087-1096, 2013.

SOUTO, R.J.C.; AFONSO, J.A.B.; MENDONÇA, C.L. et al. Achados bioquímicos, eletrolíticos e hormonais de cabras acometidas com toxemia da prenhez. Pesqui. Vet. Bras., v.33, p.1174-1182, 2013.

ULIAN, C.M.V.; FERNANDES, S.; RAMOS, P.R.R. et al. Avaliação da absorção colostral em neonatos ovinos da raça Bergamácia. Arq. Bras. Med. Vet. Zootec., v.66, p.705-712, 2014. 